\title{
Higher Levels of Cystatin C Are Associated with Worse Cognitive Function in Older Adults with Chronic Kidney Disease: The Chronic Renal Insufficiency Cohort Cognitive Study"
}

\author{
Kristine Yaffe, MD, ${ }^{a, b, c, d}$ Manjula Kurella-Tamura, MD, MPH, ${ }^{e, f}$ Lynn Ackerson, PhD, ${ }^{g}$ Tina D. \\ Hoang, MSPH, ${ }^{b}$ Amanda H. Anderson, PhD, ${ }^{i}$ Mark Duckworth, MPH, Alan S. Go, MD, ${ }^{g}$ Marie \\ Krousel-Wood, MD, MSPH, ${ }^{k, l, m}$ John W. Kusek, PhD, ${ }^{n}$ James P. Lash, MD, ${ }^{o}$ Akinlolu Ojo, MD, \\ PhD, ${ }^{p, q}$ Nancy Robinson, PhD, ${ }^{i}$ Ashwini R. Sehgal, MD, ${ }^{r, s, t}$ James H. Sondheimer, MD, ${ }^{u}$ Susan \\ Steigerwalt, MD, ${ }^{v, w}$ and Raymond R. Townsend, MD, for the CRIC Study Investigators
}

OBJECTIVES: To determine the association between cognition and levels of cystatin $\mathrm{C}$ in persons with chronic kidney disease (CKD).

DESIGN: Prospective observational study.

SETTING: Chronic Renal Insufficiency Cohort Cognitive Study.

PARTICIPANTS: Individuals with a baseline cognitive assessment completed at the same visit as serum cystatin C measurement $(\mathrm{N}=821$; mean age $64.9,50.6 \%$ male, $48.6 \%$ white).

From the ${ }^{\mathrm{a}}$ Departments of Psychiatry; ${ }^{\mathrm{b}}$ Neurology; ${ }^{\mathrm{c}}$ Epidemiology and Biostatistics, School of Medicine, University of California at San Francisco, San Francisco, California; ${ }^{\mathrm{d}}$ San Francisco Veterans Affairs Medical Center, San Francisco; ${ }^{\mathrm{e}}$ Division of Nephrology, School of Medicine, Stanford University; ${ }^{\mathrm{f}}$ Palo Alto Veterans Affairs Medical Center, Palo Alto; ${ }^{\mathrm{g} D i v i s i o n}$ of Research, Kaiser Permanente, Oakland; ${ }^{\mathrm{h}}$ Northern California Center for Research and Education, San Francisco, California; ${ }^{\mathrm{i}}$ Center for Clinical Epidemiology and Biostatistics; ${ }^{\mathrm{i}}$ Renal, Electrolyte, and Hypertension Division, Perelman School of Medicine, University of Pennsylvania, Philadelphia, Pennsylvania; Departments of ${ }^{\mathrm{k}}$ Medicine; ${ }^{\mathrm{l}}$ Epidemiology, Tulane University; ${ }^{\mathrm{m}}$ Centre for Health Research, Ochsner Clinic Foundation, New Orleans, Louisiana; ${ }^{n}$ National Institute of Diabetes and Digestive and Kidney Diseases, National Institutes of Health, Bethesda, Maryland; 'Department of Medicine, College of Medicine, University of Illinois at Chicago, Chicago, Illinois; Departments of ${ }^{\mathrm{P}}$ Internal Medicine; ${ }^{\mathrm{q}}$ Nephrology, Medical School, University of Michigan, Ann Arbor, Michigan; Departments of ${ }^{\mathrm{r}}$ Medicine; ${ }^{\mathrm{s}}$ Biomedical Ethics; ${ }^{t}$ Epidemiology and Biostatistics, Case Western Reserve University, Cleveland, Ohio; "Department of Internal Medicine, School of Medicine, Wayne State University; ' Division of Nephrology and Hypertension, Department of Medicine; and ${ }^{\mathrm{w}}$ Chronic Kidney Disease Clinic, St. John Hospital and Medical Center, Detroit, Michigan.

*The members of CRIC Study Investigators are present in Acknowledgments.

Address correspondence to Kristine Yaffe, University of California San Francisco, 4150 Clement Street, Box 181, San Francisco, CA 94121.

E-mail: kristine.yaffe@ucsf.edu

DOI: $10.1111 /$ jgs. 12986
MEASUREMENTS: Levels of serum cystatin C were categorized into tertiles; cognitive function was assessed using six neuropsychological tests. Scores on these tests were compared across tertiles of cystatin $\mathrm{C}$ using linear regression and logistic regression to examine the association between cystatin $\mathrm{C}$ level and cognitive performance (1 standard deviation difference from the mean).

RESULTS: After multivariable adjustment for age, race, education, and medical comorbidities in linear models, higher levels of cystatin C were associated with worse cognition on the modified Mini-Mental State Examination, Buschke Delayed Recall, Trail-Making Test Part (Trails) A and Part $\mathrm{B}$, and Boston Naming $(P<.05$ for all). This association remained statistically significant for Buschke Delayed Recall $(P=.01)$ and Trails A $(P=.03)$ after additional adjustment for estimated glomerular filtration rate (eGFR). The highest tertile of cystatin $\mathrm{C}$ was associated with greater likelihood of poor performance on Trails A (odds ratio $(\mathrm{OR})=2.17,95 \%$ confidence interval $(\mathrm{CI})=1.16$ 4.06), Trails $\mathrm{B}(\mathrm{OR}=1.89,95 \% \mathrm{CI}=1.09-3.27)$, and Boston Naming $(\mathrm{OR}=1.85,95 \% \mathrm{CI}=1.07-3.19)$ than the lowest tertile after multivariate adjustment in logistic models. CONCLUSION: In individuals with CKD, higher serum cystatin C levels were associated with worse cognition and greater likelihood of poor cognitive performance on attention, executive function, and naming. Cystatin $\mathrm{C}$ is a marker of cognitive impairment and may be associated with cognition independent of eGFR. J Am Geriatr Soc 62:1623-1629, 2014.

Key words: cystatin C; cognition; chronic kidney disease

Dystatin C, a protease inhibitor, has been studied as a
measure of kidney function and as a biomarker of
cognitive impairment. ${ }^{1-3}$ In a number of community-based 
cohort studies, high cystatin C levels were associated with greater risk of cognitive impairment, ${ }^{4,5}$ whereas other observational studies have reported an association between low cystatin C levels (in serum and cerebrospinal fluid) and greater risk of dementia. ${ }^{6,7}$ Cystatin $\mathrm{C}$ has also been linked to risk of cognitive impairment in genetic and neuropathological studies. A common polymorphism of the cystatin $\mathrm{C}$ gene is associated with greater risk of Alzheimer's disease $(\mathrm{AD}),{ }^{8-10}$ and cystatin $\mathrm{C}$ colocalizes with $\beta$ amyloid in the brain, especially in areas involved in $\mathrm{AD}$ pathology. ${ }^{11,12}$

Age, sex, and muscle mass affect cystatin C, as a measure of kidney function, much less than serum creatinine. ${ }^{13,14}$ Studies also suggest that cystatin $\mathrm{C}$ is a prognostic indicator of adverse health outcomes, although it is unclear whether these associations are independent of cystatin C's role as a marker of glomerular filtration rate. ${ }^{15}$ In older adults, higher levels of cystatin $\mathrm{C}$ are associated with greater risk of mortality, cardiovascular events, and poor physical function, ${ }^{4,16-18}$ and in individuals with chronic kidney disease (CKD), cystatin $\mathrm{C}$ may predict kidney decline and progression to end-stage kidney disease and identify those at risk of heart failure and mortality. ${ }^{14,19}$ Although individuals with CKD are also at high risk for cognitive decline and dementia, ${ }^{20-22}$ the potential of cystatin $\mathrm{C}$ as a marker of poor cognitive performance in this population has not been investigated.

The Chronic Renal Insufficiency Cohort (CRIC) Study, a prospective observational study, was designed to determine the association between cystatin C levels and cognitive function according to six cognitive tests in older adults with CKD and to evaluate whether this association was independent of estimated glomerular filtration rate (eGFR). It was hypothesized that higher serum cystatin C level would be associated with worse cognitive performance and that this association would remain significant even after adjusting for eGFR.

\section{METHODS}

\section{Setting}

The CRIC Cognitive (CRIC COG) Study is an ancillary study of the CRIC Study.

\section{Parent Study}

Participants enrolled in the main CRIC Study from seven clinical centers across the United States (July 1, 2003 to December 31, 2006) were aged 21 to 74 and had an eGFR of 20 to $70 \mathrm{~mL} / \mathrm{min}$ per $1.73 \mathrm{~m}^{2}$ if aged 21 to 44,20 to $60 \mathrm{~mL} / \mathrm{min}$ per $1.73 \mathrm{~m}^{2}$ if aged 45 to 64 , and 20 to $50 \mathrm{~mL} / \mathrm{min}$ per $1.73 \mathrm{~m}^{2}$ if aged 65 to 74 . Participants were considered ineligible if they were institutionalized, did not provide informed consent, had previously undergone dialysis for longer than 1 month, were diagnosed with polycystic kidney disease, had received a prior organ or bone marrow transplant, had taken immunosuppressive drugs for kidney in the past 6 months, or were currently participating in a clinical trial or an ongoing cohort study at some of the centers (the African American Study of Kidney Disease and Hypertension). Other exclusion criteria were
New York Heart Association Class III or IV heart failure, cirrhosis, human immunodeficiency infection or acquired immunodeficiency syndrome, chemotherapy for cancer within 2 years, multiple myeloma, or renal cell carcinoma. Additional details of the parent study have been previously published. ${ }^{23}$

\section{CRIC COG Study}

CRIC COG studied cognitive function in elderly adults (eligibility criteria: aged $\geq 55$ and enrolled in the main CRIC Study) with CKD. ${ }^{24}$ Participants were recruited from four of the seven clinical CRIC centers located in Philadelphia, Pennsylvania; Cleveland, Ohio; Oakland, California; and Chicago, Illinois. Of the 825 CRIC COG participants $(83.9 \%$ of those invited to participate, all English speaking), 821 completed a cognitive assessment and had cystatin $\mathrm{C}$ measurement during the same in-clinic visit of the CRIC parent study. The institutional review boards at each of the participating sites and at the University of California at San Francisco approved the study. All participants gave written informed consent.

\section{Predictor Variable}

Blood samples were collected during the main CRIC clinic visit. Samples were stored at $-80^{\circ} \mathrm{C}$, and cystatin $\mathrm{C}$ was measured at the CRIC central laboratory using a nephelometer (BN II ProSpec, Siemens Healthcare Diagnostics, Deerfield, IL) that used a particle-enhanced immunonepholometric assay (N Latex Cystatin C, Dade Behring, Inc., Deerfield, IL), with a coefficient of variation of $4.9 \% .^{25}$ To correct for drift over time when using different calibrator and reagent lots, an internal standardization for CRIC cystatin $C$ was implemented. ${ }^{26}$ Cystatin $C$ values for the 821 participants (range $0.55-6.59 \mathrm{mg} / \mathrm{L}$ ) were categorized into tertiles of low $(\leq 1.230 \mathrm{mg} / \mathrm{L})$, middle $(1.231$ $1.710 \mathrm{mg} / \mathrm{L})$, and high levels $(>1.711 \mathrm{mg} / \mathrm{L})$.

\section{Cognitive Function Measures}

Baseline cognitive testing consisted of six intervieweradministered tests in English: the Modified Mini-Mental State Examination (3MS, global cognitive function), the Trail-Making Test Part A (Trails A, attention) and B (executive function), Category Fluency (verbal fluency), the Buschke Selective Reminding Test-Delayed Recall (delayed memory), and the Boston Naming Test (naming). The 3MS is a general cognitive battery assessing orientation, concentration, language, praxis, and immediate and delayed memory. Scores on the 3MS range from 0 to 100 , with higher scores indicating better function. ${ }^{27}$ Visuospatial scanning, motor speed, executive function, and attention are all measured using Trails A and B, but Trails A is largely a test of attention in which participants draw a line between randomly placed numbered circles in sequence and Trails $B$ is primarily a test of executive function in which participants draw a line between alternating patterns of circled letters and numbers in sequence. Scores range from 0 to 300 , with lower scores (seconds) indicating better function, because they are based on the time to complete a particular task. ${ }^{28}$ Category Fluency evaluates 
verbal production, semantic memory, and language. It measures the number of animals named in a 1-minute period. Higher scores indicate better function. ${ }^{29}$ The Buschke Selective Reminding Test measures verbal memory with delayed components. Participants are read a list of 12 words and asked to recall as many as possible after one trial (immediate recall) and after a 20- to 30-minute delay (delayed recall) higher scores indicate better performance. $^{30}$ The Boston Naming Test assesses language function by asking participants to name 15 objects presented in pictures; higher scores indicate higher function. ${ }^{29}$

For each cognitive test, test-specific clinically significant poor cognitive performance was defined as a score 1 standard deviation (SD) or more below the mean for the 3MS, Verbal Fluency, Buschke Delayed Recall, and the Boston Naming tests. For Trails A and B, lower scores indicate better function, and poor cognitive performance on each test was defined as a score $1 \mathrm{SD}$ or more above the mean.

\section{Covariates}

All covariates were measured at the same visit for the parent study, as was the baseline cognitive assessment. Demographic variables included age, sex, race, Hispanic ethnicity, and education. Comorbid conditions were identified through participant self-report; laboratory test values; and use of medications for diabetes mellitus, stroke, hypertension, and coronary heart disease. Serum creatinine was calibrated to isotope dilution mass spectrometry and used to estimate GFR using the Modification of Diet in Renal Disease estimating equation. ${ }^{31}$

\section{Statistical Analyses}

To assess whether there was a linear trend in participant characteristics across cystatin $C$ tertiles, analyses of variance (ANOVAs) were used for continuous variables, along with an orthogonal polynomial contrast for test of linear trend, and the Cochran-Armitage trend test was used for categorical variables. The relationship between eGFR and cystatin $\mathrm{C}$ was examined using a Pearson correlation. An ANOVA model along with an orthogonal polynomial contrast was also used to test for a linear trend in the association between cystatin $\mathrm{C}$ tertile and cognitive function score. Models were also adjusted for age, race, education, and medical comorbidities that were significantly associated with cystatin $\mathrm{C}$ in bivariate analyses $(P<.10)$. Finally, models were also adjusted for eGFR to investigate whether the association between cystatin $\mathrm{C}$ and cognition was independent of eGFR level. An interaction between cystatin C level and age group $(<65,65-74, \geq 75)$ was also tested for.

To examine the association between cystatin C level and clinically significant poor cognitive performance, odds ratios (ORs) and $95 \%$ confidence intervals (CIs) were measured in unadjusted logistic regression models. Participants in the lowest tertile of cystatin $\mathrm{C}(\leq 1.230 \mathrm{mg} / \mathrm{L})$ served as the reference group. Models were then adjusted for age, race, education, and comorbidities. All analyses were completed using SAS version 9.1 (SAS Institute, Inc., Cary, NC), and significance testing was two-sided, with a Type I error rate of $5 \%$.

\section{RESULTS}

The 821 participants had a mean age of $64.9,50.6 \%$ were male, and $48.6 \%$ were white. Participants with higher cystatin $\mathrm{C}$ tended to be older and nonwhite and have less education $(P<.001$ for all). The prevalence of diabetes mellitus, hypertension, coronary heart disease, and stroke increased $(P<.05$ for each comparison) and eGFR decreased $(P<.001)$ with increasing level of cystatin $C$ (Table 1). The Pearson correlation between eGFR and cystatin-C was $-0.82(P<.001)$.

Participants with higher cystatin C levels had worse cognitive performance on all cognitive tests than those with intermediate and lower cystatin $\mathrm{C}$ levels in unadjusted linear regression models (Table 2). After multivariable adjustment for age, race, education, and medical

Table 1. Baseline Characteristics of 821 Participants According to Cystatin C Tertile

\begin{tabular}{|c|c|c|c|c|}
\hline Characteristic $\mathbf{N}(\%)$ or Mean (SD) & $\begin{array}{l}\text { Low }(0-1.230 \mathrm{mg} / \mathrm{L}) \\
\mathrm{n}=273\end{array}$ & $\begin{array}{l}\text { Middle (1.231-1.710 mg/L), } \\
\mathrm{n}=273\end{array}$ & $\begin{array}{l}\text { High }(>1.711 \mathrm{mg} / \mathrm{L}) \\
\mathrm{n}=275\end{array}$ & $P$-Value ${ }^{\text {a }}$ \\
\hline Age, mean \pm SD & $63.6 \pm 5.0$ & $65.8 \pm 5.9$ & $65.2 \pm 5.7$ & $<.001$ \\
\hline \multicolumn{5}{|l|}{ Age, n (\%) } \\
\hline$<65$ & $168(61.5)$ & $123(45.1)$ & $129(46.9)$ & $<.001$ \\
\hline $65-74$ & $100(36.6)$ & $128(46.9)$ & $131(47.6)$ & \\
\hline$\geq 75$ & $5(1.8)$ & $22(8.1)$ & $15(5.5)$ & \\
\hline Male, n (\%) & $142(52.0)$ & $135(49.5)$ & $138(50.2)$ & .67 \\
\hline White, n (\%) & $151(55.3)$ & $136(49.8)$ & $112(40.7)$ & $<.001$ \\
\hline$>$ High school education, n (\%) & $201(73.6)$ & $172(63.0)$ & $156(56.7)$ & $<.001$ \\
\hline Hispanic, n (\%) & $6(2.2)$ & $9(3.3)$ & $7(2.6)$ & .80 \\
\hline Diabetes mellitus, n (\%) & $93(34.1)$ & $155(56.8)$ & $176(64.0)$ & $<.001$ \\
\hline Hypertension (\%) & $223(81.7)$ & $258(94.5)$ & $267(97.1)$ & $<.001$ \\
\hline Coronary heart disease, n (\%) & $55(20.2)$ & $78(28.6)$ & $104(37.8)$ & $<.001$ \\
\hline Stroke, $n(\%)$ & $25(9.2)$ & $41(15.0)$ & $41(14.9)$ & .046 \\
\hline $\begin{array}{l}\text { Estimated glomerular filtration } \\
\text { rate, } \mathrm{mL} / \mathrm{min} \text { per } 1.73 \mathrm{~m}^{2}\end{array}$ & $54.8(8.8)$ & 41.8 (7.7) & $27.5(8.2)$ & $<.001$ \\
\hline
\end{tabular}

${ }^{\text {a }}$ For test of trend across tertiles of cystatin C. 
Table 2. Cognitive Test Scores According to Cystatin C Tertile

\begin{tabular}{|c|c|c|c|c|}
\hline Cognitive Test & $\begin{array}{c}\text { Low }(0-1.230 \mathrm{mg} / \mathrm{L}) \\
\mathrm{N}=273\end{array}$ & $\begin{array}{c}\text { Mid }(1.231-1.710 \mathrm{mg} / \mathrm{L}) \\
\mathrm{N}=273 \\
\text { Mean (SE) }\end{array}$ & $\begin{aligned} \text { High } & (>1.711 \mathrm{mg} / \mathrm{L}) \\
\mathrm{N} & =275\end{aligned}$ & $P$-Value ${ }^{\text {a }}$ \\
\hline \multicolumn{5}{|c|}{ Modified Mini-Mental State Examination } \\
\hline Unadjusted & $94.9(0.4)$ & $93.0(0.4)$ & $91.1(0.4)$ & $<.001$ \\
\hline Adjusted $^{\mathrm{b}}$ & $93.2(0.6)$ & $92.5(0.6)$ & $91.2(0.6)$ & $<.001$ \\
\hline \multicolumn{5}{|c|}{ Buschke delayed recall } \\
\hline Unadjusted & $7.9(0.2)$ & $7.4(0.2)$ & $6.9(0.2)$ & $<.001$ \\
\hline Adjusted & $7.3(0.3)$ & $7.2(0.3)$ & $6.8(0.3)$ & .02 \\
\hline \multicolumn{5}{|c|}{ Trail-Making Test part A } \\
\hline Unadjusted & $43.9(2.2)$ & $55.4(2.2)$ & $66.8(2.2)$ & $<.001$ \\
\hline Adjusted & $55.3(3.4)$ & $61.9(3.3)$ & $70.5(3.4)$ & $<.001$ \\
\hline \multicolumn{5}{|c|}{ Trail-Making Test part B } \\
\hline Unadjusted & $116.9(4.7)$ & $140.7(4.7)$ & $163.1(4.8)$ & $<.001$ \\
\hline Adjusted & $152.7(6.8)$ & $161.3(6.7)$ & $176.5(6.9)$ & $<.001$ \\
\hline \multicolumn{5}{|l|}{ Boston naming } \\
\hline Unadjusted & $14.2(0.1)$ & $13.9(0.1)$ & $13.4(0.1)$ & $<.001$ \\
\hline Adjusted & $13.9(0.2)$ & $13.8(0.2)$ & $13.4(0.2)$ & .001 \\
\hline \multicolumn{5}{|l|}{ Verbal fluency } \\
\hline Unadjusted & $18.7(0.3)$ & $17.5(0.3)$ & $17.6(0.3)$ & .01 \\
\hline Adjusted & $17.3(0.5)$ & $16.8(0.5)$ & $17.3(0.5)$ & .97 \\
\hline
\end{tabular}

${ }^{\mathrm{a}}$ For test of linear trend.

${ }^{\mathrm{b}}$ Adjusted for age, race, education, diabetes mellitus, hypertension, coronary heart disease, and stroke.

comorbidities including diabetes mellitus, hypertension, coronary heart disease, and stroke, higher cystatin C levels were associated with lower performance on the 3MS (mean (SE): high tertile, 91.2 (0.6); middle tertile, 92.5 (0.6); low tertile, 93.2 (0.6)), Buschke Delayed Recall (mean (SE): high tertile, $6.8(0.3)$; middle tertile, $7.2(0.3)$; low tertile, $7.3(0.3)$ ), Trails A (mean (SE): high tertile, 70.5 (3.4); middle tertile, 61.9 (3.3); low tertile, 55.3 (3.4)), Trails B (mean (SE): high tertile, 176.5 (6.9); middle tertile, 161.3 (6.7); low tertile, 152.7 (6.8)), and Boston Naming (mean (SE): high tertile, 13.4 (0.2); middle tertile, 13.8 (0.2); low tertile, $13.9(0.2))(P<.05$ for each comparison). The association with Verbal Fluency was no longer significant (mean (SE): high tertile, $17.3(0.5)$; middle tertile, $16.8(0.5)$; low tertile, $17.3(0.5), P=.90)$. To determine whether the association between cystatin $\mathrm{C}$ and cognition was independent of another measure of kidney function, eGFR was also adjusted for in the full multivariable-adjusted model. In these models, Trails A and Buschke Delayed Recall were still significantly associated with cystatin $\mathrm{C}(P<.05$ for each). There were no significant interactions between age group and cystatin $\mathrm{C}$ level $(P>.05$ for all $)$.

Participants in the highest tertile of cystatin C had greater risk of poor cognitive performance in unadjusted models than those in the lowest tertile based on the 3MS $(\mathrm{OR}=2.73,95 \% \quad \mathrm{CI}=1.60-4.66)$, Buschke Delayed Recall $\quad(\mathrm{OR}=1.99, \quad 95 \% \quad \mathrm{CI}=1.25-3.16), \quad$ Trails $\mathrm{A}$ $(\mathrm{OR}=3.58,95 \% \mathrm{CI}=2.03-6.31)$, Trails $\mathrm{B}(\mathrm{OR}=3.00$, $95 \% \mathrm{CI}=1.85-4.85)$, and Boston Naming $(\mathrm{OR}=2.54$, $95 \% \mathrm{CI}=1.57-4.11)$. In models adjusted for age, race, education, and comorbidities, these associations remained statistically significant for Trails A $(\mathrm{OR}=2.17,95 \%$ $\mathrm{CI}=1.16-4.06)$ and $\mathrm{B}(\mathrm{OR}=1.89,95 \% \mathrm{CI}=1.07-3.27)$, and Boston Naming $(\mathrm{OR}=1.85,95 \% \mathrm{CI}=1.07-3.19)$ (Figure 1).

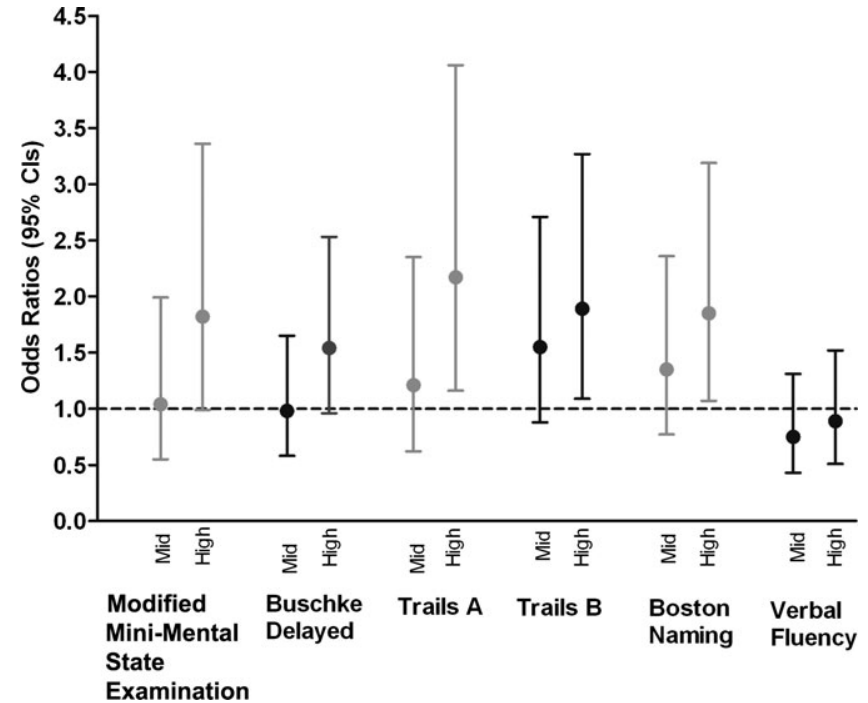

Figure 1. Adjusted odds ratios and $95 \%$ confidence intervals for poor cognitive performance across cystatin $\mathrm{C}$ tertile (reference low). Models adjusted for age, race, education, diabetes mellitus, hypertension, coronary heart disease, and stroke. Trails $=$ Trail-Making Test Part.

\section{CONCLUSION}

In a group of older adults with CKD enrolled in a prospective cohort study, a significant association was found between higher cystatin $\mathrm{C}$ levels and worse cognitive function for most cognitive domains, including global cognition, delayed memory, attention, executive function, and naming. Participants in the highest tertile of cystatin $\mathrm{C}$ were almost two times as likely to have significant poor cognitive performance on attention, executive function, and naming as those in the lowest tertile. Cystatin $\mathrm{C}$ was 
associated with attention and delayed memory after adjusting for eGFR. There was no difference in the relationship between cystatin $\mathrm{C}$ level and cognitive function according to age group.

Several studies have demonstrated that elderly adults with impaired kidney function have a greater risk of cognitive impairment and dementia based on measures of serum creatinine. ${ }^{32-34}$ The current findings of an association between cystatin $\mathrm{C}$ and cognitive function are consistent with these previous studies and provide additional evidence of a risk relationship between kidney function and cognitive impairment. The reported associations also parallel previous findings from the CRIC COG study that described associations between eGFR according to the Modification of Diet in Renal Disease and worse performance on multiple cognitive domains, including global cognition, executive function, naming, attention, and delayed memory. ${ }^{24}$

The results of this study are also consistent with those of longitudinal cohort studies of community-dwelling older adults that found an association between high cystatin C levels and incident cognitive decline as well as cognitive and physical disability. ${ }^{4,5}$ High cystatin $\mathrm{C}$ levels have also been associated with subclinical brain infarcts, ${ }^{35,36}$ and in a recent cohort study of older adults, markers of kidney function, including high cystatin $\mathrm{C}$ levels, were associated with poor global cognition and greater white matter deficits in the anterior limb of the internal capsulem, ${ }^{37}$ a region associated with impairment on executive function and episodic memory, ${ }^{38}$ although the current results are in contrast to those of other studies that support cystatin C's relationship with cognitive function but suggest that lower cystatin C levels correspond with greater risk of poor cognitive outcomes, including incident $\mathrm{AD}$ in a cohort of older men, conversion to $\mathrm{AD}$ in individuals with mild cognitive impairment, and dementia in individuals with Lewy body disease. ${ }^{6,7,39}$ These conflicting reports may be due to differences in study populations, study design, outcome definitions, or methods used to measure cystatin C.

The cross-sectional association between cystatin $\mathrm{C}$ and cognitive function in this study could be related to vascular pathology and shared pathways with cardiovascular outcomes also linked to cystatin C, including hypertension, obesity, and coronary heart disease. ${ }^{3,40-42}$ The current results also suggest a significant relationship between cystatin $\mathrm{C}$ and attention as well as delayed memory, even after adjusting for eGFR, which suggests that cystatin $C$ could affect the pathological changes found in $\mathrm{AD}$ through pathways not associated with kidney function. Several lines of evidence also support a role for cystatin $\mathrm{C}$ in neurodegenerative pathways involving amyloid plaque formation, as demonstrated by its colocalization with $\beta$-amyloid in brain tissue $^{11}$ and a high correlation with beta-amyloid and tau levels in cerebrospinal fluid. ${ }^{43}$ In animal models, cystatin C knockout mice have lower levels of soluble beta-amyloid, low plaque formation, and lower cognitive deficits, ${ }^{44}$ suggesting that higher cystatin $\mathrm{C}$ levels may increase $\mathrm{AD}$ pathology, although some in vitro and in vivo studies indicate that cystatin $\mathrm{C}$ is neuroprotective and binds directly to beta-amyloid to prevent plaque formation and preserve cognition. ${ }^{1,45,46}$
A number of studies indicate that cystatin $\mathrm{C}$ is a reliable marker for risk stratification in elderly adults with and without CKD, which may be attributable to its role as a sensitive measure of kidney function or to its relationships with non-GFR determinants such as inflammation. ${ }^{14-17,47,48}$ The current results suggest a possible relationship between cystatin $\mathrm{C}$ and worse cognition independent of eGFR levels. In earlier investigations of the association between cystatin $\mathrm{C}$ and cognitive function, studies that accounted for eGFR, by stratification or adjustment, reported that cystatin $\mathrm{C}$ remained significantly associated with cognitive impairment, although these cohorts focused on healthy older adults. ${ }^{5,6}$ The current findings suggest that, in those with $\mathrm{CKD}$, cystatin $\mathrm{C}$ is complementary to creatinine-based eGFR in its association with cognitive function, but it may also provide additional prognostic value. The observed association between cystatin $\mathrm{C}$ and specific cognitive domains could be related to non-GFR determinants of cystatin C.

The current study had a number of strengths, including participant recruitment from the ongoing multicenter CRIC Study, which comprises a large cohort of diverse individuals. As a result, it was possible to evaluate cognitive performance on a variety of cognitive tests. In addition, CRIC participants are well characterized, with complete data on many participant characteristics, including sociodemographic variables and cardiovascular comorbidities, allowing for adjustment for several potential covariates and confounders as well as level of kidney function.

These results should be interpreted in light of the study limitations. This was a cross-sectional analysis, so causality cannot be inferred. In addition, data were not available on structural brain imaging or pathological results to determine the etiology of clinically significant poor cognitive performance. Finally, these findings may not be generalizable to younger populations because the CRIC COG cohort was composed of older adults.

This study found that high cystatin C levels were associated with greater likelihood of poor cognitive performance in older adults with CKD, and for some domains, this association was independent of eGFR levels. Although the effect sizes were small, and the findings are preliminary, the findings suggest a need for further investigation to determine the relationship between cystatin $C$ levels and cognitive outcomes in healthy individuals and those with CKD, as well as for additional longitudinal studies to determine the potential role for cystatin $\mathrm{C}$ as a biomarker for cognitive decline.

\section{ACKNOWLEDGMENTS}

CRIC Study Investigators: Lawrence J. Appel, MD, MPH, Harold I. Feldman, MD, MSCE, Alan S. Go, MD, Jiang $\mathrm{He}, \mathrm{MD}, \mathrm{PhD}$, John W. Kusek, PhD, James P. Lash, MD, Akinlolu Ojo, MD, PhD, Mahboob Rahman, MD, and Raymond R. Townsend, MD.

Conflict of Interest: Dr. Yaffe is a consultant for Novartis and serves on data safety monitoring boards for Takeda, Inc. and a study sponsored by the National Institutes of Health (NIH) and on the Beeson Scientific Advisory Board. 
Funding for the CRIC Study was obtained under a cooperative agreement from the National Institute of Diabetes and Digestive and Kidney Diseases (U01DK060990, U01DK060984, U01DK061022, U01DK061021, U01DK 061028, U01DK060980, U01DK060963, and U01DK 060902). This work was also supported in part by the Perelman School of Medicine at the University of Pennsylvania (Clinical and Translational Science Award NIH/ NCATS UL1TR000003), Johns Hopkins University (UL1 TR-000424), the University of Maryland (GCRC M01 RR-16500), the Clinical and Translational Science Collaborative of Cleveland, UL1TR000439 from the National Center for Advancing Translational Sciences component of the NIH and NIH Roadmap for Medical Research, the Michigan Institute for Clinical and Health Research (UL1TR000433), the University of Illinois at Chicago (CTSA UL1RR029879), Tulane University Translational Research in Hypertension and Renal Biology (P30GM 103337), Kaiser NIH/NCRR (UCSF-CTSI UL1 RR024131). The CRIC COG ancillary study is supported by National Institute of Diabetes and Digestive and Kidney Diseases Grant R01DK069406.

Author Contributions: Study concept and design: Yaffe. Acquisition of subjects and data: Yaffe, KurellaTamura, Duckworth, Go, Kusek, Lash, Ojo, Robinson, Townsend. Analysis and interpretation of data: Yaffe, Ackerson. Preparation of manuscript: Yaffe, KurellaTamura, Ackerson, Hoang, Anderson, Duckworth, Go, Krousel-Wood, Kusek, Lash, Ojo, Robinson, Sehgal, Sondheimer, Steigerwalt, Townsend.

Sponsor's Role: The study sponsors did not participate in the design, methods, subject recruitment, data collections, analysis, or preparation of the paper.

\section{REFERENCES}

1. Kaeser SA, Herzig MC, Coomaraswamy J et al. Cystatin C modulates cerebral [beta]-amyloidosis. Nat Genet 2007;39:1437-1439.

2. Kaur G, Levy E. Cystatin C in Alzheimer's disease. Front Mol Neurosci 2012;5:79.

3. Bugnicourt J-M, Godefroy O, Chillon J-M et al. Cognitive disorders and dementia in CKD: The neglected kidney-brain axis. J Am Soc Nephrol 2013;24:353-363

4. Sarnak MJ, Katz R, Fried LF et al. Cystatin C and aging success. Arch Intern Med 2008;168:147-153.

5. Yaffe K, Lindquist K, Shlipak MG et al. Cystatin-C as a marker of cognitive function in elders: Findings from the Health ABC Study. Ann Neurol 2008;63:798-802.

6. Sundelof J, Arnlov J, Ingelsson E et al. Serum cystatin C and the risk of Alzheimer disease in elderly men. Neurology 2008;71:10721079.

7. Maetzler W, Schmid B, Synofzik M et al. The CST3 BB genotype and low cystatin C cerebrospinal fluid levels are associated with dementia in Lewy body disease. J Alzheimers Dis 2010;19:937-942.

8. Cathcart HM, Huang R, Lanham IS et al. Cystatin C as a risk factor for Alzheimer disease. Neurology 2005;64:755-757.

9. Finckh U, von der Kammer H, Velden J et al. Genetic association of a cystatin $\mathrm{C}$ gene polymorphism with late-onset Alzheimer disease. Arch Neurol 2000;57:1579-1583.

10. Hua Y, Zhao H, Lu X et al. Meta-Analysis of the cystatin C (CST3) gene G73A Polymorphism and susceptibility to Alzheimer's disease. Int J Neurosci $2012 ; 122: 431-438$.

11. Deng A, Irizarry MC, Nitsch RM et al. Elevation of Cystatin C in susceptible neurons in Alzheimer's disease. Am J Pathol 2001;159:10611068.

12. Levy EP, Sastre MP, Kumar AP et al. Codeposition of cystatin C with amyloid-[beta] protein in the brain of Alzheimer disease patients. J Neuropathol Exp Neurol 2001;60:94-104
13. Coll E, Botey A, Alvarez L et al. Serum cystatin C as a new marker for noninvasive estimation of glomerular filtration rate and as a marker for early renal impairment. Am J Kidney Dis 2000;36:29-34.

14. Menon V, Shlipak MG, Wang X et al. Cystatin C as a risk factor for outcomes in chronic kidney disease. Ann Intern Med 2007;147:19-27.

15. Tangri N, Inker LA, Tighiouart $\mathrm{H}$ et al. Filtration markers may have prognostic value independent of glomerular filtration rate. J Am Soc Nephrol 2012;23:351-359.

16. Shlipak MG, Katz R, Sarnak MJ et al. Cystatin C and prognosis for cardiovascular and kidney outcomes in elderly persons without chronic kidney disease. Ann Intern Med 2006;145:237-246.

17. Shlipak MG, Sarnak MJ, Katz R et al. Cystatin C and the risk of death and cardiovascular events among elderly persons. N Engl J Med 2005;352:2049-2060

18. Peralta CA, Shlipak MG, Judd S et al. Detection of chronic kidney disease with creatinine, cystatin $\mathrm{C}$, and urine albumin-to-creatinine ratio and association with progression to end-stage renal disease and mortality. JAMA 2011;305:1545-1552.

19. Bhavsar NA, Appel LJ, Kusek JW et al. Comparison of measured GFR, serum creatinine, cystatin C, and beta-trace protein to predict ESRD in African Americans with hypertensive CKD. Am J Kidney Dis 2011;58:886-893.

20. Kurella M, Chertow GM, Fried LF et al. Chronic kidney disease and cognitive impairment in the elderly: The Health, Aging, and Body Composition Study. J Am Soc Nephrol 2005;16:2127-2133.

21. Kurella M, Chertow GM, Luan J et al. Cognitive impairment in chronic kidney disease. J Am Geriatr Soc 2004;52:1863-1869.

22. Kurella M, Yaffe K, Shlipak MG et al. Chronic kidney disease and cognitive impairment in menopausal women. Am J Kidney Dis 2005;45:66-76.

23. Feldman HI, Appel LJ, Chertow GM et al. The Chronic Renal Insufficiency Cohort (CRIC) Study: Design and methods. J Am Soc Nephrol 2003;14: S148-S153.

24. Yaffe K, Ackerson L, Tamura MK et al. Chronic kidney disease and cognitive function in older adults: Findings from the Chronic Renal Insufficiency Cohort Cognitive Study. J Am Geriatr Soc 2010;58:338-345.

25. Erlandsen EJ, Randers E, Kristensen JH. Evaluation of the Dade Behring N Latex Cystatin C assay on the Dade Behring Nephelometer II System. Scand J Clin Lab Invest 1999;59:1-8.

26. Anderson A, Xie D, Tao $\mathrm{K}$ et al. Internal standardization of cystatin C measurements in the Chronic Renal Insufficiency Cohort (CRIC) Study. J Am Soc Nephrol 2010;21:176A. ASN abstract 287.

27. Teng EL, Chui HC. The modified Mini-Mental State (3MS) Examination. J Clin Psychiatry 1987;48:314-318.

28. Reitan RM. Validity of the Trail Making Test as an indicator of organic brain damage. Percept Mot Skills 1958;8:271-276.

29. Welsh KA, Butters N, Mohs RC et al. The Consortium to Establish a Registry for Alzheimer's Disease (CERAD). Part V. A normative study of the neuropsychological battery. Neurology 1994;44:609-614.

30. Buschke H, Fuld PA. Evaluating storage, retention, and retrieval in disordered memory and learning. Neurology 1974;24:1019-1025.

31. Coresh J, Astor BC, McQuillan G et al. Calibration and random variation of the serum creatinine assay as critical elements of using equations to estimate glomerular filtration rate. Am J Kidney Dis 2002;39:920-929.

32. Seliger SL, Siscovick DS, Stehman-Breen CO et al. Moderate renal impairment and risk of dementia among older adults: The Cardiovascular Health Cognition Study. J Am Soc Nephrol 2004;15:1904-1911.

33. Slinin Y, Paudel ML, Ishani A et al. Kidney function and cognitive performance and decline in older men. J Am Geriatr Soc 2008;56:2082-2088.

34. Tamura MK, Wadley V, Yaffe $\mathrm{K}$ et al. Kidney function and cognitive impairment in US adults: The Reasons for Geographic and Racial Differences in Stroke (REGARDS) study. Am J Kidney Dis 2008;52:227-234.

35. Seliger SL, Longstreth WT Jr, Katz R et al. Cystatin C and subclinical brain infarction. J Am Soc Nephrol 2005;16:3721-3727.

36. Wada M, Nagasawa H, Kawanami $\mathrm{T}$ et al. Cystatin $\mathrm{C}$ as an index of cerebral small vessel disease: Results of a cross-sectional study in communitybased Japanese elderly. Eur J Neurol 2010;17:383-390.

37. Rajagopalan P, Refsum H, Hua X et al. Mapping creatinine- and cystatin C-related white matter brain deficits in the elderly. Neurobiol Aging 2013;34:1221-1230.

38. Smith EE, Salat DH, Jeng J et al. Correlations between MRI white matter lesion location and executive function and episodic memory. Neurology 2011;76:1492-1499.

39. Ghidoni R, Benussi L, Glionna M et al. Plasma cystatin C and risk of developing Alzheimer's disease in subjects with mild cognitive impairment. J Alzheimers Dis 2010;22:985-991.

40. Kestenbaum B, Rudser KD, de Boer IH et al. Differences in kidney function and incident hypertension: The Multi-Ethnic Study of Atherosclerosis. Ann Intern Med 2008;148:501-508. 
41. Muntner P, Winston J, Uribarri J et al. Overweight, obesity, and elevated serum cystatin C levels in adults in the United States. Am J Med 2008;121: 341-348.

42. Gr Luc, Bard J-M, Cl Lesueur et al. Plasma cystatin-C and development of coronary heart disease: The PRIME Study. Atherosclerosis 2006;185:375-380.

43. Sundelof J, Sundstrom J, Hansson O et al. Cystatin C levels are positively correlated with both a-beta 42 and tau levels in cerebrospinal fluid in persons with Alzheimer's disease, mild cognitive impairment, and healthy controls. J Alzheimers Dis 2010;21:471-478.

44. Sun B, Zhou Y, Halabisky B et al. Cystatin C-cathepsin B axis regulates amyloid beta levels and associated neuronal deficits in an animal model of Alzheimer's disease. Neuron 2008;60:247-257.
45. Tizon B, Ribe EM, Mi W et al. Cystatin C protects neuronal cells from amyloid-beta-induced toxicity. J Alzheimers Dis 2010;19:885894.

46. Sastre M, Calero M, Pawlik M et al. Binding of cystatin C to Alzheimer's amyloid $\beta$ inhibits in vitro amyloid fibril formation. Neurobiol Aging 2004;25:1033-1043.

47. Shlipak MG, Wassel Fyr CL, Chertow GM et al. Cystatin C and mortality risk in the elderly: The Health, Aging, and Body Composition Study. J Am Soc Nephrol 2006;17:254-261.

48. Peralta CA, Katz R, Sarnak MJ et al. Cystatin C identifies chronic kidney disease patients at higher risk for complications. J Am Soc Nephrol 2011;22:147-155. 